\title{
Safe eye surgery: non-technical aspects
}

\begin{abstract}
The traditional training of surgeons focused exclusively on developing knowledge, clinical expertise, and technical (surgical) skills. However, analyses of the reasons for adverse events in surgery have revealed that many underlying causes originate from behavioural or non-technical aspects of performance (eg, poor communication among members of the surgical team) rather than from a lack of surgical (ie, technical) skills. Therefore, technical skills appear to be necessary but not sufficient to ensure patient safety. Paying attention to non-technical skills, such as team working, leadership, situation awareness, decision making, and communication, will increase the likelihood of maintaining high levels of error-free performance. Identification and training of non-technical skills has been developed for high-risk careers, such as civil aviation and nuclear power. Only recently, training in non-technical skills has been adopted by the surgical world and anaesthetists. Non-technical skills need to be tailored to the environment where they are used, and eye surgery has some substantial differences compared with other surgical areas, for example, high volume of surgery, use of local anaesthetics, and very sophisticated equipment. This review highlights the need for identification of the non-technical skills relevant to eye surgeons and promotion of their use in the training of eye surgeons. Eye (2011) 25, 1109-1111; doi:10.1038/eye.2011.127; published online 3 June 2011
\end{abstract}

Keywords: surgery; complications; education; training; safety

Patients in the United Kingdom undergo more than eight million surgical procedures per year, and ophthalmology represents a substantial proportion of the overall surgical volume (eg, over 300000 cataract surgeries per year). But surgery is risky and safety practices are
A Azuara-Blanco ${ }^{1}$, A Reddy², G Wilkinson ${ }^{3}$ and R Flin ${ }^{3}$

inconsistent. The Reporting and Learning System (RLS of the National Patient Safety Agency) recorded 446184 reports of patient safety incidents from surgical specialties between 1 January 2005 and 30 September 2008. ${ }^{1}$ Of these, $15311(3.4 \%)$ were in ophthalmology. ${ }^{1}$ The real number of incidents is probably greater. The nature of these incidents is hugely varied, from wrong site surgery to misplaced patient notes. Although many of these incidents are not serious, some have led to patient harm.

Studies into medical error show that it is frequently 'behavioural' aspects of performance that are causal factors rather than individuals not having the necessary technical expertise. ${ }^{2}$ These aspects of performance can be described as non-technical skills (following from European civil aviation), as they relate to cognitive and social skills necessary for safe and effective practice in a clinical context, that complement technical skills. ${ }^{3}$ Thus, they address a whole range of behaviours that support good performance, such as team working, task management, situation awareness, and decision making. Current data are compelling. Breakdowns in team working and communication, lack of situation awareness, and poor decision making can lead to poor outcomes for surgical patients. ${ }^{4,5}$ Communication was found to be a causal factor in $43 \%$ of errors made in surgery. ${ }^{6}$ In another study, cognitive and diagnostic errors in the operating room contributed to $27 \%$ of claims against a health-care organisation. ${ }^{7}$ Among surgical specialties, ophthalmology occupied the first place in surgical errors due to wrong intraocular lens implantation. ${ }^{8}$ The second most common error in ophthalmic surgery is administering local anaesthetic to the wrong eye. Errors including operating in the wrong site/wrong patient/procedure are rare but unacceptable for the public. ${ }^{8}$

The medical profession increasingly recognises that there is more to good surgical
${ }^{1}$ Health Services Research Unit, University of Aberdeen, Aberdeen, UK

${ }^{2}$ Aberdeen Royal Infirmary, The Eye Clinic, Aberdeen, UK

${ }^{3}$ School of Psychology, University of Aberdeen, King's College, Old Aberdeen, UK

Correspondence: A Azuara-Blanco, Health Services Research Unit, University of Aberdeen, 3rd Floor, Health Sciences Building, Foresterhill, Aberdeen, AB25 2ZD, UK Tel: + 44 (0) 1224 552730; Fax: + 44 (0) 1224554580 E-mail: aazblanco@ abdn.ac.uk

Received: 18 January 2011 Accepted in revised form: 14 April 2011 Published online: 3 June 2011 
practice than having extensive clinical knowledge and good technical and surgical skills. However, as mentioned above, aspects such as decision making, leadership, and team working provide a vital underpinning for effective performance. We propose that behavioural areas of competence should receive explicit attention during ophthalmic surgical training to ensure that individuals are aware of what constitutes good practice and have the requisite skills to deliver this. Recognition of the importance of these non-technical skills in anaesthesia has led to the development of aviation-style crew resource management training courses to address them, ${ }^{5}$ for example, Crisis Avoidance and Resource Management, ${ }^{2}$ and Anaesthesia Crisis Resource Management. ${ }^{9}$ A taxonomy of anaesthetists' non-technical skills and a behavioural scale to rate them are now being used in some anaesthetic training programmes. ${ }^{10}$ For surgeons, a non-technical skills for surgeons (NOTSS) taxonomy and rating system was developed. ${ }^{11,12}$ The system was developed using task analysis with subject matter experts and evaluated in trials using standardised video scenarios and real operations. It allows consultant (attending) surgeons to give feedback to colleagues and trainees based on structured observations of non-technical aspects of performance during intraoperative surgery. Training in NOTSS is offered by The Royal College of Surgeons of Edinburgh, and is likely to be introduced as a part of performance-based assessment for UK surgeons. It is being used in a number of countries, and the Royal Australasian College of Surgeons have incorporated NOTSS into their new professional standards. ${ }^{13}$

Although the Royal College of Ophthalmologists has valuable guidelines on promoting patient safety, ${ }^{14}$ no specific training in non-technical skills is currently offered. Each working environment has its own unique non-technical skill requirements. Although there are a lot of similarities between eye surgery and other surgical specialties, some differences are worth noting.

1. High volume and high turnover increase the potential for errors. Cataract surgery is the most common operation in the NHS. A possible mistake would be to use a wrong IOL. Other high-volume interventions include retinal laser photocoagulation and intraocular injections of anti-VEGF therapies. Injecting anti-VEGF in a wrong eye is an error likely to happen if there are no solid systems, as elderly patients may be confused and with bilateral age-related macular degeneration.

2. Most commonly, surgery is performed under local anaesthesia and with the patient awake. Special attention to verbal communication among team members in the operative environment is needed. For example, if a complication occurs, or if a manoeuvre by a trainee needs to be corrected, interaction between the surgeons and other members of the team needs to take into account that the patient is aware of the conversations.

3. Current day-case practice lessens the opportunity for patient-doctor interaction. One-stop surgery practised in many units is even more challenging because of time constraints and the potential for overlooking serious medical morbidity in patients that can lead to an intraoperative adverse event. The discussion of risk and benefits of surgery often is done by other staff not involved in the surgery, and the surgeon may not be aware of patient expectations.

4. Heavily reliant on very specialised equipment (eg, phacoemulsification and laser). The surgical team needs to have a clear understanding of what needs to be calibrated and checked, when, and what to do when the equipment fails.

5. Most patients are elderly with concomitant medical conditions and thus vulnerable to dangers of surgery. For example, using $\alpha 1$-blockers and patients on warfarin may increase the surgical risk.

The principal non-technical skills categories may be generic and relatively transferable across domains, but the component behaviours that demonstrate proficiency in those skills within a particular environment (eg, the operating room for ophthalmic surgery) are specific to the needs and characteristics of the domain. The greatest challenge in developing non-technical skills training in eye surgery will be to identify explicitly the competences that are necessary for good practice and hence need to be trained. It is then essential to be able to describe these skills in sufficient detail to allow them to be included in the syllabus and then assessed reliably during and following the training. Techniques such as cognitive task analysis are very effective for this and as mentioned above have been successfully used in the medical field in identifying the non-technical competencies needed by anaesthetists and surgeons.

Once a list of non-technical competencies has been produced, this can be used as the basis for syllabus development, supporting debriefing and, most importantly, can be used as an assessment tool for evaluating individual trainees' non-technical skills and the overall effectiveness of training. Assessment tools of this type (called behavioural marker systems as they rely on observation of behaviour to indicate level of skill) can be defined as 'observable, non-technical behaviours that contribute to superior or substandard performance' ${ }^{15}$ For effective non-technical skills assessment, the 
behavioural marker systems used must be explicit, transparent, and above all validated, and assessors must be trained to ensure the systems are used reliably.

In summary, further research is needed on the non-technical skill proficiencies required for safe ophthalmic surgery and to describe them in sufficient detail so they can be taught and reliably assessed. Ideally both non-technical and technical aspects of training should be integrated for achieving the safest possible practice.

\section{Conflict of interest}

The authors declare no conflict of interest.

\section{References}

1 Catchpole K, Panesar SS, Russell J, Tang V, Hibbert P, Cleary K. National Patient Safety Agency 2009, National Reporting and Learning Service, Surgical safety can be improved through better understanding of incidents. Reference: 1116. Available online at http://www.nrls.npsa. nhs.uk/resources/type/data-reports/?entryid $45=63054$. Last accessed on 24 July 2010.

2 Fletcher GC, McGeorge P, Flin RH, Glavin RJ, Maran NJ. The role of non-technical skills in anaesthesia: a review of current literature. $\mathrm{Br} J$ Anaesth 2002; 88: 418-429.

3 Flin R, O'Connor P, Crichton M. Safety at the Sharp End: A Guide to Non-Technical Skills. Farnham: Ashgate, 2008.

4 de Leval MR, Carthey J, Wright DJ, Farewell VT, Reason JT. Human factors and cardiac surgery: a multicenter study. J Thorac Cardiovasc Surg 2000; 119: 661-672.
5 Helmreich RL, Davies JM. Anaesthetic simulation and lessons to be learned from aviation. Can J Anaesth 1997; 44: 907-912.

6 Gawandee AA, Zinner MJ, Studdert DM, Brennan TA. Analysis of errors reported by surgeons at three teaching hospitals. Surgery 2003; 133: 614-621.

7 Wilson J. A practical guide to risk management in surgery: developing and planning. Health Care Risk Resources. International - Royal College of Surgeons Symposium: Edinburgh, 1999.

8 Simon JW, Ngo Y, Khan S, Strogatz D. Surgical confusions in ophthalmology. Arch Ophthalmol 2007; 125: 1515-1522.

9 Fletcher G, McGeorge P, Flin R, Glavin RJ, Maran NJ. The role of non-technical skills in anaesthesia: a review of current literature. Br J Anaesth 2002; 88: 418-429.

10 Flin R, Patey R, Glavin R Maran N. Anaesthetists' Non-Technical skills (ANTS). Br J Anaesth 2010; 105: 38-44.

11 Yule S, Flin R, Paterson-Brown S, Maran N, Rowley D. Development of a rating system for surgeons' non-technical skills. Med Educ 2006; 40: 1098-1104.

12 Yule S, Flin R, Maran N, Rowley D, Youngson G, Paterson-Brown S. Surgeons' non-technical skills in the operating room: reliability testing of the NOTSS behavior rating system. World J Surg 2008; 32: 548-556.

13 Dickinson I, Watters D, Graham I, Montgomery P, Collins J. Guide to the assessment of competence and performance in practising surgeons. ANZ J Surg 2009; 79: 198-204.

14 Kelly SP. Royal College of Ophthalmologists. Guidance on patient safety in ophthalmology from the Royal College of Ophthalmologists. Eye 2009; 23: 2143-2151.

15 Klampfer B, Flin R, Helmreich RL, Hausler R, Sexton B, Fletcher $\mathrm{G}$ et al. Enhancing Performance in High Risk Environments: Recommendations for the use of Behavioural Markers. Ladenburg: Daimler-Benz Shiftung, 2001. Downloadable version available from: www.psyc.abdn. ac.uk/serv02. Last accessed on 12 September 2010. 